Voix et Images

\title{
Jacques Pelletier, Lecture politique du roman québécois contemporain
}

\section{Robert Major}

Volume 11, numéro 3 (33), printemps 1986

Yolande Villemaire

URI : https://id.erudit.org/iderudit/200588ar

DOI : https://doi.org/10.7202/200588ar

Aller au sommaire du numéro

Éditeur(s)

Université du Québec à Montréal

ISSN

0318-9201 (imprimé)

1705-933X (numérique)

Découvrir la revue

Citer cet article

Major, R. (1986). Jacques Pelletier, Lecture politique du roman québécois contemporain. Voix et Images, 11(3), 543-547. https://doi.org/10.7202/200588ar d'utilisation que vous pouvez consulter en ligne.

https://apropos.erudit.org/fr/usagers/politique-dutilisation/ 
CHRONIQUES 543

\title{
Jacques Pelletier, Lecture politique du roman québécois contemporain ${ }^{1}$.
}

\author{
par Robert Major, Université d'Ottawa.
}

Ce livre, le premier des "Cahiers du département d'études littéraires" de l'UQAM, a été rapidement suivi par trois autres cahiers (le Social et le littéraire (Anthologie), Travaux sémiotiques, Sémiologies), respectivement signés ou préparés par Jacques Pelletier, Jacques Allard et Gilles Thérien, tous membres du comité de lecture de la collection. Force est de reconnaitre que les animateurs de celle-ci n'ont pas pris à la légère la promesse de publier au moins trois titres par année; et que pour diffuser toute recherche susceptible de faire avancer notre connaissance du phénomène littéraire (la prétention à la "modernité» est tombée après le premier cahier) ils ont eux-mêmes pris les choses en main. Il y a quelque chose de réjouissant dans ce dynamisme des départements de littérature qui cherchent, souvent par des publications rapidement fabriquées (trop rapidement peut-être: celle-ci est en train de se défaire sous mes mains pourtant déférentes!), à combler les retards, les lacunes ou tout simplement les silences des revues ou collections "officielles", manifestant ainsi leur sens pratique et leur souci, intellectuel et pédagogique, de produire avec célérité des instruments de référence et de travail. Quatre titres en un an, c'est mieux que la plupart des revues - qui paient ainsi le prix d'un appareil souvent très lourd - et c'est amplement suffisant pour attiser notre intérêt. Qu'en est-il donc de ce premier cahier?

Le volume de Jacques Pelletier est un recueil d'études toutes publiées antérieurement, la plupart ici même dans Voix et images, entre 1970 et 1983. Double emploi donc (triple même, dans le cas du chapitre II), mais qui ne saurait nous surprendre et encore moins nous scandaliser puisqu'il a acquis ses lettres de noblesse depuis longtemps: ces reprises sont en quelque sorte consubstantielles au métier d'écrivain ou de critique. Ici et là on publie quelques articles, quelques livres. Vient un jour où l'on interroge ces signes de soi (J.-L. Major, le Jeu en étoile, 1976, p. 10). On se relit, moins pour établir un bilan qu'un tracé (loc.cit.). Et ce tracé, nouvellement révélé par la relecture d'essais dispersés au cours des ans et des circonstances, surprend quelquefois le signataire même des textes. Jacques Pelletier fait écho à cette révélation de soi à soi dès les premiers mots de sa préface:

En relisant les études que j'ai consacrées au roman québécois contemporain depuis une dizaine d'années, il m'est apparu évident qu'elles relevaient toutes, à des degrés d'intensité variable, d'une même 
préoccupation fondamentale: dégager l'enjeu - culturel, social, politique - impliqué dans ces productions. Cette évidence que je. pratiquais une lecture politique ou, pour reprendre une expression de Pierre Barberis, une lecture idéologique des textes, je ne l'ai bien sûr pas toujours possédée.

Le signataire décide donc d'assumer le tracé révélé en republiant ses traces et le lecteur est devant un livre, avec son unité et son orientation propres.

Dans ce cas-ci, un livre composé de sept chapitres et d'une post-face. Le premier chapitre pose le nationalisme comme horizon idéologique le plus englobant de la littérature québécoise: nationalisme défensif jusqu'au début des années 1960, puis offensif (décolonisateur), comme le révèlent bien les romans d'Aquin, de Godbout ou même de Ducharme. Les chapitres suivants étudient successivement les romans de Jacques Godbout, André Major, Victor-Lévy Beaulieu, Jacques Ferron et Paul Villeneuve. Le dernier chapitre, en scrutant quelques œuvres particulières d'auteurs moins connus et plus récents (Gilles Raymond, Yvon Paré, Pierre Filion), s'interroge sur la renaissance possible du roman social, sur le retour à la grande tradition classique du roman (réaliste et engagé: engagé parce que réaliste) après vingt ans de formalisme: retour qui ne saurait déplaire à l'auteur.

Ces chapitres sont d'inégale longueur, les trois plus importants (trente pages ou plus) étant consacrés à Godbout, Major et Beaulieu; en regard de ces études, les chapitres sur Paul Villeneuve ( 5 p.) ou sur les "romans sociaux" ( 7 p.) ont davantage le caractère de notes de lecture: d'intelligents résumés qui dégagent sommairement l'importance relative de ces œuvres. Les chapitres, aussi, sont inégalement réussis, même si chacun présente un indéniable intérêt et même si tous se lisent avec plaisir et profit. Plaisir: car la prose de Pelletier est claire, précise, sans prétention, dénuée des tics à la mode et, en particulier, peu contaminée par les clichés des milieux de gauche dont il se réclame pourtant; profit: car il est lecteur perspicace et curieux et un observateur attentif (et compétent) des réalités politiques québécoises. Chaque chapitre pourrait même être présenté comme modèle de parcours méthodique: l'introduction pose nettement les problèmes qui préoccupent l'auteur et ses hypothèses de recherche; les divisions de l'étude sont claires, les charnières nombreuses et explicites, l'argumentation transparente et sans détours (quitte à abuser des répétitions et des j'y reviendrai!); la conclusion présente donc tout naturellement sa synthèse et une coda sans équivoque. On peut être en désaccord avec Pelletier à chacune des étapes de son parcours; mais on ne peut guère lui reprocher de manquer de méthode et de franchise.

Ceci dit, certaines études restent plus convaincantes que d'autres. Ainsi, l'analyse du parcours romanesque de Jacques Godbout me semble le plus réussi des chapitres. Car les autres, malgré leur intérêt évident, laissent songeur.

L'étude sur Jacques Ferron, par exemple, explique les changements apportés à la Nuit (1965) - qui deviendra les Confitures de coings (1972) - 
par la vision très nette qu'avait Ferron de la crise d'Octobre 1970 (la majuscule est de Pelletier), dont il avait été un témoin passionné et un acteur privilégié. La présentation est systématique et convaincante. Mais outre que ces transformations avaient déjà été étudiées et que Ferron lui-même avait déjà signalé leur signification essentielle dans son «Appendice aux Confitures de coings....", demeure la question (non soulevée et à plus forte raison non résolue) qui, dans l'esprit du lecteur naïf, devrait être l'aboutissement normal de cette présentation de l'influence de la politique sur la littérature: quel en a été l'effet littéraire? Il y a eu modification; l'œuvre a été "corrigée» ou remaniée. Il y a eu effet scriptural, en quelque sorte. Mais l'effet littéraire? Or, ce ne serait pas insulter la mémoire de Jacques Ferron, il me semble, que de reconnaître que ces modifications font injure à ce petit chef-d'œuvre qu'est la Nuit. La littérature a perdu au change. L'influence d'Octobre 1970, i.e. de la politique sur la littérature, elle est là aussi; elle est là surtout, dans l'esprit d'un littéraire: à trop vouloir signifier, à trop marquer sa "tendance " (pour parodier Engels), un écrivain superbement intelligent et extraordinairement doué (mais aussi rancunier et souvent injuste, reconnaissons-le) gâte lui-même sans doute le plus parfait de ses récits. Leçon terrible et sans doute salutaire qu'il aurait été opportun, il me semble, de soulever, si ce n'est qu'en conclusion.

Des interrogations quelque peu différentes sont soulevées par les autres études (sur Major et Beaulieu, en particulier). Dans le cas de Ferron, l'événement ou la situation politique, littérairement déterminante, est présentée de façon tout à fait probante par Pelletier. Mais la relation alternativement établie entre la politique et les cuvres de Major et de Beaulieu ne me semble guère aussi concluante. Le passage des œuvres à la conjoncture se fait plutôt allègrement, presque magiquement. Que la trilogie d'André Major soit l'expression de la stagnation sous le régime Bourassa par un compagnon de route du Parti Québécois (p. 75); que l'œuvre de V.-L. Beaulieu traduise le profond désarroi de la petite bourgeoisie intellectuelle qui, d'un côté, refuse la société dominante et ses fausses valeurs mais qui, de l'autre, ne conçoit d'autre solution qu'individuelle (p. 99), le lecteur veut bien. Mais ici, comme toujours, se soulève le spectre de toutes les lectures de type sociocritique: comment passer de la constatation d'un parallélisme entre l'univers imaginaire et la vie collective à la mise en relation rigoureuse de ces deux mondes? Pelletier est bien conscient de ce problème crucial des médiations. C'est ainsi, par exemple - pour le contourner! - qu'il présentera l'étude sur Beaulieu comme une simple sociologie des contenus, une simple étude de la société telle qu'elle est représentée dans le texte (p. 84), reconnaissant explicitement les difficultés rencontrées lorsqu'on veut expliquer (en termes goldmanniens), c'est-à-dire insérer l'œuvre dans la structure englobante et déterminante. Mais il ne s'en tiendra pas à son programme, car constamment ces romans sont perçus comme symptomatiques du désarroi de toute une classe (sans que soit posé ou réglé le problème de la classe à laquelle appartiendrait Beaulieu!). Le plus souvent, toutefois, plutôt que d'éviter le problème (et de s'y buter malgré tout!), Pelletier fera appel à des textes autobiographiques où l'écrivain pose explicitement sa relation à l'histoire; le critique se sent alors justifié 
d'approfondir la relation postulée, l'écrivain ayant précisé qu'il a telle perception de l'acte d'écriture ou de la société québécoise ou que tel roman a été composé dans telle situation. Mais la question reste entière: est-ce parce que l'écrivain affirme une relation qu'il y en a nécessairement une? Et cette relation a-t-elle nécessairement la signification perçue par l'écrivain? Il y a bien longtemps - et pour ne s'en tenir qu'au domaine de la sociocritique qu'a été posé le dilemme de la «conscience fausse» de l'écrivain, c'est-à-dire de l'écrivain qui se trompe radicalement sur-la signification idéologique objective de son œuvre. Qu'a été posé aussi le paradoxe de "l'écume» et du "courant profond » qu'affectionnait bien Lénine et qui sera repris par tous les épigones. Bourassa, telle grève, le P. Q.... est-ce de l'ordre de l'écume ou du courant profond? Qu'en est-il de la relation établie?

Certes, il ne saurait être question de reprocher à Pelletier l'absence d'une théorie des médiations dans son petit livre et tel n'est pas le sens de mes réserves. D'ailleurs, la critique d'inspiration socio-historique dans son ensemble est encore loin d'une telle théorie. La critique marxiste, en particulier, a multiplié depuis un siècle les tentatives (théorie du reflet, théorie de la vision du monde, théorie de l'idéologie, théories sociocritiques à caractère sémiologique), toutes stimulantes mais encore problématiques. Henri Mitterand peut bien regretter qu'on ne se soit jamais aventuré à mettre en faisceau les facteurs qui surdéterminent la parole d'un romancier (le Discours du roman, 1980, p. 10), mais quelle entreprise herculéenne ce serait de le faire. Démêler cet écheveau, pour un seul écrivain, et à un seul moment de sa carrière est une tâche qu'on ne peut exiger d'un seul homme, fût-il Sartre. Il ne s'agit donc pas de reprocher à Pelletier ses ellipses, ses silences ou ses court-circuits entre œuvre et société (court-circuit: mise en relation de deux points à potentiel différent par un conducteur de faible résistance...); il s'agit d'en prendre acte tout simplement. Cette constatation, d'abord, assure en partie la qualité heuristique de ce petit livre (sa vertu stimulante); elle permet ensuite de comprendre pourquoi dans ce recueil l'étude des romans de Godbout est plus satisfaisante que les autres. Il s'avère que cette étude est plus longue, plus touffue, plus détaillée, donc plus précise dans son analyse des ouvres et de la conjoncture; il s'avère surtout qu'elle semble plus consciente des différents niveaux ou champs à l'intérieur de cette conjoncture, Pelletier distinguant les strates littéraire, idéologique et politique comme niveaux successifs d'insertion de l'œuvre. Les "points" ayant été multipliés, les court-circuits (inévitables!) sont moins perceptibles (moins "choquants"!). Certes, Ezra Pound qui proposait (Comment lire, 1928) que l'on jette dehors tous les critiques qui utilisent des termes vagues et généraux, aurait peut-être mal réagi face à une étude où l'œuvre littéraire est tour à tour perçue comme un témoignage sur... ou un écho de..., une expression de... ou une traduction de..., un révélateur de... ou une mise en forme de..., déterminée par une conjoncture ou s'inscrivant dans un cadre, entretenant des rapports de similitude ou bien d'homologie avec la réalité sociale..., toutes notions interchangeables. Mais dans l'état présent des études littéraires, comment éviter complètement ces ambiguïtés? Ces notions sont les plus précises dans les circonstances, à moins de faire œuvre théorique, ce qui n'est manifestement pas le but de Pelletier. 
Mais quel est son but, au fait? La question n'est pas oiseuse si l'on songe que le titre de son livre inscrit d'emblée celui-ci dans une famille précise de titres et dans une communauté de critiques (Barberis, Leenhardt, Prévost, Macherey ..., avec Goldmann, Lukacs et l'ombre de Lénine - Lénine faisant une lecture "politique" de Tolstoï - à l'arrière-plan), pour qui la critique littéraire est une dimension importante de la lutte idéologique. Or, dans sa "Préface», Pelletier insistera justement sur son expérience de militant politique et sur son incapacité à percevoir la littérature comme pures aventures du langage: son activité de critique est prolongement et illustration de son activité militante. Ainsi, sa lecture politique n'est pas seulement tentative académique de saisir les relations entre des ceuvres littéraires et la politique au sens large; elle est effort pour déterminer l'efficace propre de ces ouvres comme interventions dans l'histoire. D'où, vraisemblablement, le choix des écrivains étudiés: nationalistes, gauchisants ou sceptiques devant le vouloirvivre collectif de leurs compatriotes. D'où, vraisemblablement aussi, l'existence de cette "Postface» ( «Notes pour un auto-portrait politique d'un intellectuel petit-bourgeois des années $60 \mathrm{~m}$ ) qui me semblait, aux premières lectures, plutôt gratuite (et quelque peu complaisante, soyons honnête), mais dont la justification, à la réflexion, me paraît double: présenter l'activité critique comme tributaire d'un engagement plus profond; montrer que pour qu'il y ait sociocritique (l'œuvre, produit de l'histoire, agissant historiquement et s'expliquant par sa réinsertion dans l'histoire), il y a nécessité impérieuse de préciser aussi la situation du critique dans l'échiquier social. La critique doit aussi, doit simultanément, être auto-critique.

I. Jacques Pelletier, Lecture politique du roman québécois contemporain; essais, Montréal, Université du Québec à Montréal, 1984, 150 p. 